\title{
Sodium Gradient: A Tool to Individualize Dialysate Sodium Prescription in Chronic Hemodialysis Patients?
}

\author{
E. Lars Penne ${ }^{a, b}$ Olga Sergeyeva ${ }^{b}$ \\ ${ }^{a}$ VU University Medical Center, Amsterdam, The Netherlands; ${ }^{\text {b }}$ Renal Research Institute, New York, N.Y., USA
}

\section{Key Words}

Sodium gradient $\cdot$ Dialysate sodium concentration •

Serum sodium concentration $\cdot$ Intradialytic symptoms

\begin{abstract}
Low dialysate sodium concentrations have been associated with intradialytic symptoms such as muscle cramps and hypotensive episodes. High dialysate sodium concentrations lead to sodium loading, thirst and subsequent increase in interdialytic weight gain and hypertension. The optimal dialysate sodium concentration for an individual depends on the serum sodium concentration. The difference between the dialysate sodium concentration and the predialysis serum sodium concentration has been defined as the sodium gradient. In this article, the role of the sodium gradient in fluid overload, hypertension, intradialytic symptoms and clinical outcome is discussed. Absolute serum sodium levels should always be taken into account when interpreting the relation between sodium gradient and clinical outcomes. Alignment of the dialysate sodium with the serum sodium concentration may be beneficial in many patients.
\end{abstract}

Copyright $\odot 2011$ S. Karger AG, Basel

\section{Introduction}

The dialysate sodium concentration has been gradually increased since the early years of hemodialysis treatment to permit high ultrafiltration volumes as dialysis times decreased without compromising treatment tolerance [1]. Until the introduction of controlled ultrafiltration in the 1970s, dextrose-containing hyponatremic dialysis fluids were prescribed to facilitate water and sodium removal by osmotic and diffusive gradients [1]. Although nowadays most of the excess sodium is removed by ultrafiltration, diffusive sodium removal still has an important clinical impact on the total intradialytic sodium balance [2, 3]. High dialysate sodium levels have been advocated to reduce intradialytic hypotensive episodes and symptoms such as cramping and nausea [4], but are associated with fluid overload and hypertension $[3,5]$, which may in the long term lead to left ventricular hypertrophy and cardiovascular events. Low dialysate concentrations have been related to improved volume and blood pressure control, but may lead to more intradialytic symptoms in some patients [6].

The optimal dialysate sodium concentration is different for each individual and largely depends on the sodium concentration gradient, i.e. the difference between

Lars Penne, MD, $\mathrm{PhD}$

Department of Nephrology

VU University Medical Cente

Boelelaan 1117, NL-1081 HV Amsterdam (The Netherlands)

Tel. +31 20444 307, Fax +31 20444 313, E-Mail el.penne@vumc.nl 


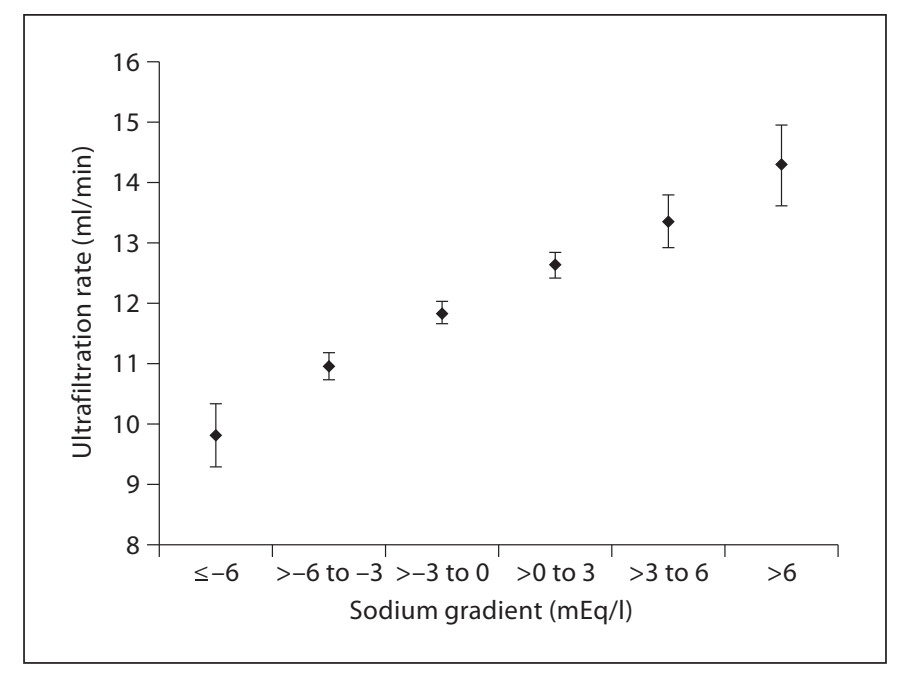

Fig. 1. Relation between sodium gradient and ultrafiltration rate. Error bars represent 95\% CI. Ultrafiltration rate was adjusted for sex, age, race and body weight.

the dialysate and the predialysis serum sodium concentration [7]. The serum sodium concentration is generally stable over time in hemodialysis patients $[3,8]$. If the dialysate sodium concentration is higher than the predialysis serum sodium (i.e. a positive gradient), there is a diffusive flux of sodium over the membrane from the dialysate to the blood compartment, which will attenuate the total intradialytic sodium removal and may cause net sodium loading. Conversely, a dialysate sodium concentration lower than the predialysis serum sodium (i.e. a negative gradient) is characterized by diffusive transport of sodium from the blood to the dialysate and contributes to sodium removal and possible deficiency.

Sodium gradient is a potentially useful tool to individualize the dialysate sodium prescription [9]. In the present article, the relation between sodium gradient and short- and long-term clinical outcomes is reviewed and recommendations are provided on how the dialysate sodium prescription could be adjusted based on the sodium gradient.

\section{Sodium Gradient, Intradialytic Weight Gain and Blood Pressure}

It has been postulated that each individual has an inherent plasma sodium concentration or 'sodium setpoint' $[3,7]$. Hence, each increase in the serum sodium concentration as a result of dietary intake or sodium administration during hemodialysis treatment (e.g. a positive sodium gradient) is followed by a thirst-driven water intake to reduce the osmolarity until the corresponding serum sodium concentration is reached. A strong association has been demonstrated between thirst and intradialytic weight gain [10].

Increased thirst and water intake in patients with a positive gradient require higher ultrafiltration rates in order to remove this excess fluid during the next hemodialysis session. We have previously analyzed the relation between the sodium gradient and ultrafiltration rate in a retrospective cohort of chronic in-center hemodialysis patients receiving dialysis at Renal Research Institute (RRI) clinics [ $n=4,128$; age $61 \pm 15$ (SD); $54 \%$ males; $55 \%$ blacks]. As shown in figure 1, we found a clear relationship between the sodium gradient and the ultrafiltration rate, indicating higher interdialytic weight gain in patients with a positive sodium gradient as reported previously [3]. If an ultrafiltration rate of more than $5 \%$ of the total body weight would be considered as a crude indicator of fluid overload, then patients with a positive gradient $(>0 \mathrm{mEq} / \mathrm{l})$ would be much more likely to be fluid overloaded than patients with a negative or zero gradient [odds ratio $(\mathrm{OR})=2.7 ; \mathrm{p}<0.001$ ]. In our data, we found no clinically relevant relationship between sodium gradient and blood pressure level, possibly because of differences in antihypertensive therapy. While a positive sodium gradient is related to sodium loading, high weight gain and increased blood pressure levels, lowering the sodium gradient may contribute to improved blood pressure control. Years ago, Murisasco et al. [11] aligned the dialysate sodium level with the patients' sodium 'equilibrium point'. The equilibrium point was defined as the dialysate concentration at which no decrease or increase in the plasma sodium during treatment was seen. Applying this approach to 65 chronic hemodialysis patients substantially lowered the blood pressure and reduced the number of patients on antihypertensive drugs from 19 (29\%) to 6 patients (9\%). In a more recent study, the dialysate sodium level was individualized in 27 stable patients. The dialysate sodium concentration was on average reduced by $3 \mathrm{mEq} / \mathrm{l}$ in order to achieve a sodium gradient of $0 \mathrm{mEq} / \mathrm{l}$. After 3 weeks of follow-up, interdialytic weight gain was decreased, thirst scores had improved, and in the subgroup of patients with uncontrolled hypertension blood pressure levels were reduced [12]. Several other interventional studies have investigated the effects of an absolute reduction in dialysate sodium concentration - without taking into account the gradient - 
on intradialytic weight gain and blood pressure level, as reviewed by Santos and Peixoto [6]. Most of these studies reported reductions in intradialytic weight gain and blood pressure level after reducing the dialysate sodium concentration. In summary, most data consistently show a strong relation between dialysate sodium level, weight gain and blood pressure level. Notably, there are no data from interventional studies investigating whether a reduction in the dialysate sodium level below the serum sodium level is beneficial in overhydrated and/or hypertensive hemodialysis patients.

\section{Sodium Gradient and Intradialytic Symptoms}

Many factors play a role in the pathogenesis of intradialytic hypotension. However, its occurrence is rare in the absence of high ultrafiltration rates [13]. In view of the strong relationship between sodium gradient, intradialytic weight gain and ultrafiltration rate, we hypothesized that patients with a positive gradient are at an increased risk of intradialytic symptoms. We therefore investigated the relationship between the sodium gradient and the risk of intradialytic morbid events in the same cohort as mentioned above, consisting of 4,128 prevalent hemodialysis patients from RRI clinics [14]. For this analysis, we retrieved records of all saline infusion during treatment from the database. If more saline was administered than needed for priming and rinsing of the extracorporeal circuit, this treatment was classified as one with an intradialytic morbid event. Subsequently, patients were divided into quartiles according to the percentage of treatments with intradialytic morbid events. Intradialytic morbid events occurred in 42,404 of 186,287 treatments (23\%). Logistic regression was applied to calculate the risk of frequent intradialytic morbid events [i.e. $\geq 32 \%$ (upper quartile) of treatments complicated with an intradialytic morbid event] in groups of patients with different sodium gradients. After adjustment for patient characteristics and ultrafiltration rate, the risk of frequent intradialytic morbid events was increased in patients with a positive sodium gradient $[\mathrm{OR}=1.3(95 \% \mathrm{CI} 0.97-1.8, \mathrm{p}=0.08)$ for a sodium gradient between +3 and $+6 \mathrm{mEq} / \mathrm{l}$, and $\mathrm{OR}=$ $3.3(95 \%$ CI $2.2-4.9, \mathrm{p}<0.0001)$ for a gradient $>+6 \mathrm{mEq} / \mathrm{l}$; fig. 2]. The relation between gradient and hypotensive episodes was independent of the ultrafiltration rate, which might be explained by low serum sodium levels in patients with chronic heart failure or by direct effects of sodium on hemodynamic stability. Besides, it has been suggested previously that sodium itself is a uremic toxin

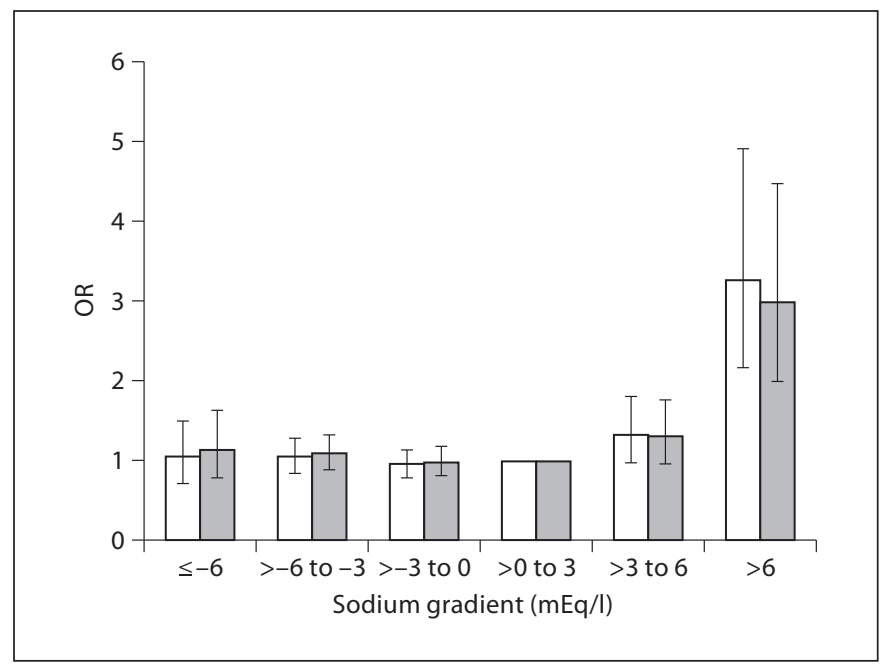

Fig. 2. Hazard ratios of frequent intradialytic morbid events, as defined by saline infusions in $\geq 32 \%$ of the treatments, divided by sodium gradient. Sodium gradient $0-3 \mathrm{mEq} / \mathrm{l}$ is the reference group. Columns in white represent crude OR; columns in grey represent OR after adjustments for sex, age, vintage, diabetes and ultrafiltration rate normalized for body weight. Error bars represent 95\% CI.

[15]. Although our analysis was limited by its observational character, a positive sodium gradient was identified as a novel and potentially modifiable risk factor for intradialytic morbid events.

In line with these findings, a reduction in the dialysate sodium level based on the serum sodium level resulted in fewer hypotensive episodes and intradialytic symptoms in previous intervention studies $[11,12]$. One study demonstrated significant reductions in hypotensive episodes from 9 to $2 \%$ after reducing the dialysate sodium level from 138 to a mean value of $135 \mathrm{mEq} / \mathrm{l}$, depending on the individual predialysis serum sodium level [12]. Others reported almost complete disappearance of intradialytic symptoms after establishing an individualized sodium prescription [11]. It is likely that a lower sodium gradient in these patients resulted in less thirst, less fluid overload and therefore less need for removal of excess fluid. In contrast, in another study a reduction in the dialysate sodium concentration from 140 to $135 \mathrm{mEq} / \mathrm{l}$ resulted in a substantial rise of intradialytic episodes from 17 to $55 \%$ of the treatments [16]. However, in the latter study the average serum sodium decreased by $6 \mathrm{mEq} /$ lover the course of the treatment, indicating that the dialysate sodium concentration must have been much lower than the predialysis serum sodium in most patients. These patients 
Table 1. Proposed adjustments of the dialysate sodium prescription according to the sodium gradient in patients with and without frequent intradialytic symptoms

\begin{tabular}{llll}
\hline Gradient & $\begin{array}{l}\text { Intradialytic Recommendation } \\
\text { symptoms }\end{array}$ & Background \\
\hline Positive & No & $\begin{array}{l}\text { Align dialysate with serum sodium } \\
\text { concentration }\end{array}$ & $\begin{array}{l}\text { Prevent sodium loading } \\
\text { Improved fluid and blood pressure control } \\
\text { Positive gradient related with increased hospitalization and } \\
\text { mortality rates }\end{array}$ \\
\hline Positive & Yes & $\begin{array}{l}\text { Align dialysate with serum sodium } \\
\text { concentration }\end{array}$ & $\begin{array}{l}\text { Lowering dialysate sodium leads to reduction in symptoms, as long } \\
\text { as dialysate sodium is not lower than serum sodium concentration }\end{array}$ \\
\hline Negative & No & $\begin{array}{l}\text { Consider increase in dialysate sodium } \\
\text { in patients with large negative gradient }\end{array}$ & $\begin{array}{l}\text { Trend suggesting an association between steep negative gradient } \\
\text { and mortality } \\
\text { Theoretical increased activation of RAAS and SNS }\end{array}$ \\
\hline Negative & Yes & $\begin{array}{l}\text { Align dialysate with serum sodium } \\
\text { concentration }\end{array}$ & \begin{tabular}{l} 
Increasing dialysate sodium may reduce intradialytic symptoms \\
\hline
\end{tabular} \\
\hline
\end{tabular}

RAAS = Renin-angiotensin-aldosterone system; SNS = sympathetic nervous system.

were thus dialyzed against a steep negative sodium gradient, which could well be the explanation for the observed increase in number of symptoms.

It should be mentioned that the effect of sodium profiles on intradialytic symptoms remains not fully elucidated. It has been suggested that potential benefits of sodium profiling can largely be explained by high time averaged dialysate sodium concentrations [17].

Taken together, these data suggest that a decrease in the dialysate sodium concentration contributes to improved tolerability of dialysis treatment, as long as the dialysate sodium concentration is not substantially lower than the predialysis serum sodium concentration. Alignment of the dialysate sodium with the serum sodium concentration may be the preferable strategy to minimize intradialytic symptoms.

\section{Sodium Gradient and Long-Term Clinical Outcomes}

There are limited data linking the sodium gradient with long-term clinical outcomes. Previously, we have shown that a sodium gradient $>+3 \mathrm{mEq} / \mathrm{l}$ was associated with increased hospitalization and mortality in more than 4,000 incident hemodialysis patients [18]. This was explained by chronic exposure to a positive sodium balance during hemodialysis treatment, resulting in volume overload and all its consequences. Interestingly, mortality rates also tended to increase in patients with a strong negative sodium gradient [unpubl. obs.]. The latter finding might be explained by volume depletion during treatment, which may contribute to hypotension and potentially harmful overactivation of the sympathetic nervous system [19]. Alternatively, the high serum sodium level in these patients may reflect dehydration and comorbid conditions and explain the mortality risk. Prospective studies investigating dialysate sodium concentrations on clinical endpoints are therefore indicated.

\section{What Is the Optimal Dialysate Sodium Concentration?}

In patients with a positive sodium gradient, a lower dialysate sodium concentration should be considered to prevent sodium loading and reduce thirst. As a consequence, intradialytic weight gain will typically decrease and blood pressure control may improve. Moreover, a lower dialysate sodium concentration in these patients can reduce the number of intradialytic hypotensive episodes.

In patients with a negative sodium gradient, an increase in the sodium prescription should be considered for patients with frequent intradialytic symptoms. The optimal dialysate sodium concentration for patients with a negative gradient without intradialytic symptoms is unclear. Since a negative sodium gradient can be conceptualized as a form of sodium restriction, it is reasonable to 
argue that a more liberate sodium-restricted diet in patients with negative sodium gradients could be justified. This may especially be beneficial to patients who have difficulties to comply with dietary recommendations. On the other hand, as mentioned above, a very large negative gradient may in the long-term also be harmful. Notably, both positive and negative sodium gradients are associated with abnormal serum sodium levels and might be indicative of comorbidity. Our recommendations how to change the dialysate sodium concentration for patients with and without frequent intradialytic symptoms are summarized in table 1 .

\section{Limitations for the Use of Sodium Gradient in Clinical Practice}

Sodium gradient is dependent on serum sodium concentration. In other words, patients with a high positive gradient are more likely to have a low serum sodium concentration, accompanying comorbid conditions such as congestive heart failure. Patients with a negative gradient are more likely to have a high serum sodium concentration, which may, for instance, reflect dehydration. Moreover, the serum sodium concentration may vary over time in some patients, for instance in poorly controlled diabetics [20]. This limits the interpretation of our observational data. Prospective interventional studies are therefore warranted to investigate the effects of a sodium alignment strategy as proposed above, stratified for patients with low or high serum sodium levels.

Apart from the above-mentioned aspects, alignment of the dialysate with serum sodium concentration using standard laboratory measurements lacks precision. Small measurement errors are generally irrelevant in standard clinical practice, but may become significant for the purpose of sodium alignment in hemodialysis patients. Moreover, the measured sodium concentration does not necessarily reflect the amount of sodium that is available for diffusion through the dialyzer membrane. On the one hand, the active sodium concentration is higher than the reported sodium concentration, since sodium is only distributed in the plasma water [21]. On the other hand, a fraction of the active sodium is bound to (negatively charged) proteins and is thus unavailable for diffusion (i.e. Gibbs-Donnan effect) $[1,6]$. In general, these opposing effects tend to cancel out [22]. Finally, the preparation of the dialysate may lack precision and dialysis machines may not be adequately calibrated. Although these inaccuracies in the measurement and delivery of sodium lim- it the efforts to align the dialysate sodium with the serum sodium concentration, they can be partly overcome by using the average of consecutive sodium measurements, rather than just one single value. Alternatively, dialysate and plasma conductivity measurements have been proposed to individualize the dialysate composition. Plasma conductivity measurements can be used as a tool to increase intradialytic sodium removal [23]. However, they do not actually measure pre-and post-sodium levels.

\section{Conclusions}

Sodium gradient is a potentially important modifiable factor to improve treatment tolerability and prevent unnecessary sodium loading in chronic hemodialysis patients. Especially in patients with frequent intradialytic symptoms, adjustments of the dialysate sodium concentration can be beneficial. In an ongoing randomized controlled trial (NCT01015313) at the RRI in New York, the dialysate sodium concentration is aligned with the serum sodium as part of a multifactorial approach to restrict sodium in hemodialysis patients. This study will hopefully provide valuable data regarding the long-term effects of an individualized dialysate sodium prescription.

\section{Acknowledgements}

We kindly thank Peter Kotanko and Nathan Levin for their valuable comments and suggestions for improvement of the manuscript. We are also grateful to Jochen Raimann, Stephan Thijssen and Len Usvyat for their contribution in data analyses and supportive discussions regarding the sodium gradient. Lars Penne received a research grant from the Dutch Kidney Foundation.

\section{Disclosure Statement}

The authors declare no conflict of interest.

References

1 Flanigan MJ: Role of sodium in hemodialysis. Kidney Int Suppl 2000;76:S72-S78.

-2 Mann H, Stiller S: Sodium modeling. Kidney Int Suppl 2000;76:S79-S88.

3 Keen ML, Gotch FA: The association of the sodium 'setpoint' to interdialytic weight gain and blood pressure in hemodialysis patients. Int J Artif Organs 2007;30:971-979.

-4 Henrich WL, Woodard TD, McPhaul JJ Jr: The chronic efficacy and safety of high sodium dialysate: double-blind, crossover study. Am J Kidney Dis 1982;2:349-353. 
5 Gotch FA, Lam MA, Prowitt M, Keen M: Preliminary clinical results with sodiumvolume modeling of hemodialysis therapy. Proc Clin Dial Transplant Forum 1980;10: 12-17.

6 Santos SF, Peixoto AJ: Revisiting the dialysate sodium prescription as a tool for better blood pressure and interdialytic weight gain management in hemodialysis patients. Clin J Am Soc Nephrol 2008;3:522-530.

$\checkmark 7$ Levin NW, Zhu F, Keen M: Interdialytic weight gain and dry weight. Blood Purif 2001;19:217-221.

$\checkmark 8$ Peixoto AJ, Gowda N, Parikh CR, Santos SF: Long-term stability of serum sodium in hemodialysis patients. Blood Purif 2010;29: 264-267.

$\checkmark 9$ Penne EL, Levin NW, Kotanko P: Improving volume status by comprehensive dietary and dialytic sodium management in chronic hemodialysis patients. Blood Purif 2010;30:7178.

10 Bots CP, Brand HS, Veerman EC, et al: Interdialytic weight gain in patients on hemodialysis is associated with dry mouth and thirst. Kidney Int 2004;66:1662-1668.

-11 Murisasco A, France G, Leblond G, et al: Sequential sodium therapy allows correction of sodium-volume balance and reduces morbidity. Clin Nephrol 1985;24:201-208.
12 de Paula FM, Peixoto AJ, Pinto LV, Dorigo D, Patricio PJ, Santos SF: Clinical consequences of an individualized dialysate sodium prescription in hemodialysis patients. Kidney Int 2004;66:1232-1238.

13 Sherman RA: Intradialytic hypotension: an overview of recent, unresolved and overlooked issues. Semin Dial 2002;15:141-143.

14 Penne EL, Usvyat L, Raimann JG, et al: Intradialytic sodium gradient is a potential modifiable risk factor for intradialytic morbid events in chronic hemodialysis (abstract); XLVII ERA-EDTA Congr, Munich, June 2010.

15 Ritz E, Dikow R, Morath C, Schwenger V: Salt - a potential 'uremic toxin'? Blood Purif 2006;24:63-66.

16 Ozturk S, Taymez DG, Bahat G, et al: The influence of low dialysate sodium and glucose concentration on volume distributions in body compartments after haemodialysis: a bioimpedance analysis study. Nephrol Dial Transplant 2008;23:3629-3634.

17 Song JH, Park GH, Lee SY, Lee SW, Lee SW, Kim MJ: Effect of sodium balance and the combination of ultrafiltration profile during sodium profiling hemodialysis on the maintenance of the quality of dialysis and sodium and fluid balances. J Am Soc Nephrol 2005; 16:237-246.

18 Sergeyeva O, Usvyat L, Kotanko P, Levin NW: Positive intradialytic sodium gradients relate to intradialytic weight gain, hospitalization rate, and mortality in chronic hemodialysis patients (abstract). NDT Plus 2009; 2:ii650.
19 Zoccali C, Mallamaci F, Parlongo S, et al: Plasma norepinephrine predicts survival and incident cardiovascular events in patients with end-stage renal disease. Circulation 2002;105:1354-1359.

20 Penne EL, Thijssen S, Raimann JG, Levin NW, Kotanko P: Correction of serum sodium for glucose concentration in hemodialysis patients with poor glucose control. Diabetes Care 2010;33:e91.

-21 Burnett RW, Covington AK, Fogh-Andersen $\mathrm{N}$, et al: Recommendations for measurement of and conventions for reporting sodium and potassium by ion-selective electrodes in undiluted serum, plasma or whole blood. International Federation of Clinical Chemistry and Laboratory Medicine (IFCC). IFCC Scientific Division Working Group on Selective Electrodes. Clin Chem Lab Med 2000;38: 1065-1071.

22 Kimura G, Van Stone JC, Bauer JH, Keshaviah PR: A simulation study on transcellular fluid shifts induced by hemodialysis. Kidney Int 1983;24:542-548.

23 Manlucu J, Gallo K, Heidenheim PA, Lindsay RM: Lowering postdialysis plasma sodium (conductivity) to increase sodium removal in volume-expanded hemodialysis patients: a pilot study using a biofeedback software system. Am J Kidney Dis 2010;56: 69-76. 